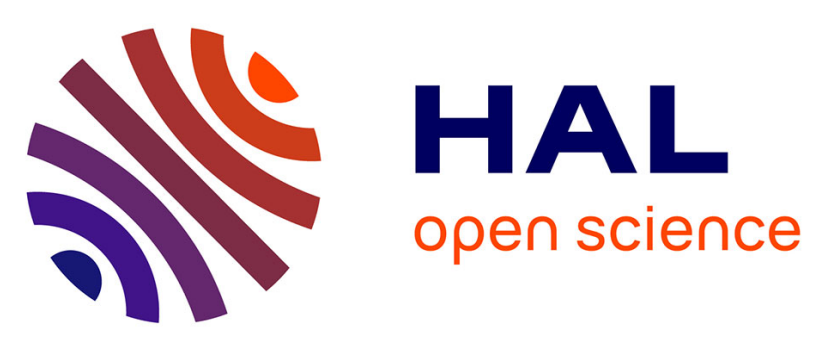

\title{
A pressure based method for vaporizing compressible two-phase flows with interface capturing approach
}

Benjamin Duret, R. Canu, J. Reveillon, F.X. Demoulin

\section{To cite this version:}

Benjamin Duret, R. Canu, J. Reveillon, F.X. Demoulin. A pressure based method for vaporizing compressible two-phase flows with interface capturing approach. International Journal of Multiphase Flow, 2018, 108, pp.42-50. 10.1016/j.ijmultiphaseflow.2018.06.022 . hal-01827180

\author{
HAL Id: hal-01827180 \\ https://hal.science/hal-01827180
}

Submitted on 13 Sep 2018

HAL is a multi-disciplinary open access archive for the deposit and dissemination of scientific research documents, whether they are published or not. The documents may come from teaching and research institutions in France or abroad, or from public or private research centers.
L'archive ouverte pluridisciplinaire HAL, est destinée au dépôt et à la diffusion de documents scientifiques de niveau recherche, publiés ou non, émanant des établissements d'enseignement et de recherche français ou étrangers, des laboratoires publics ou privés. 


\title{
A pressure based method for vaporizing compressible two-phase flows with interface capturing approach
}

\author{
B. Duret*, R. Canu, J. Reveillon, F.X. Demoulin \\ CORIA-UMR 6614 - Normandie Université, CNRS-Université et INSA de Rouen, Campus \\ Universitaire du Madrillet, 76800 Saint Etienne du Rouvray, France.
}

\begin{abstract}
This study focuses on the development of a pressure based method able to capture compressibility effects and phase change in turbulent two-phase flow DNS, using the Coupled Level Set/Volume of Fluid (CLSVOF) interface capturing method. A compressible VOF equation and a new pressure equation are described, including additional terms due to vaporization and compressibility. First, validation cases without phase change such as a gas-water shock tube and an oscillating water column configuration have been performed. Concerning the first case, obtained results suggests that the method is able to capture accurately the shock wave characteristics. For the second case, a reference solution is computed and compared with DNS results, showing the method accuracy and convergence.

Finally, a two-phase Homogeneous Isotropic Turbulence with compressibility and phase change is investigated. Contrary to previous works, the vaporization process has an impact on the flows dynamics. The effects of the vaporization on the gas density is illustrated and compared with a reference solution for a constant vaporization rate. Great agreement is obtained between DNS results and the reference solution. The mass balance between total mass, gas and liquid mass is also shown and demonstrates the efficiency of the CLSVOF interface capturing method regarding mass conservation, even in a turbulent atomization context.
\end{abstract}

*Corresponding author : duret@coria.fr

Preprint submitted to International Journal of Multiphase Flow

September 13, 2018 
Keywords: Compressible, DNS, interface, Two-phase flows, vaporization

\section{Introduction}

Compressible or weakly compressible liquid-gas flows occur in many fields such as liquid fuel injection, plunging waves, cavitation, sloshing, drowning damaged nuclear reactor, phase change heat transfer, pipeline of two-phase flows,

5 etc. Liquid-gas flows has a direct impact on gas emissions (atomization in combustion engines), industrial process efficiency (heat exchanger) or coastal engineering (breaking wave). Most numerical simulations dedicated to the aforementioned applications use an incompressible formalism, which does not take into account the compressibility effects and density variation among each phase. However, compressibility can have a significant effect in a wide range of configuration, from liquid jet injection (cavitation inside an injector) to breaking wave configuration (impact of the entrained air or bubbles). Indeed, vapor production or cavitation inside an injector generate a creation of volume (non-zero divergence of the velocity field) incompatible with the standard incompress-

15 ible hypothesis used mainly in liquid-gas flows simulations. In the framework of violent impact of two-phase flows as for example in an impinging liquid jet or a wave breaking process, it is well known that the presence of bubbles or entrained air close to the wall/structure has important consequences on both the flow, the structure and even the environment through the propagation of sound. In the framework of marine energy development, it has been shown that the violent expansion/compression of the gas due to acoustic wave propagation, which can also develop in shock waves in most dramatic cases, has a strong impact on the incoming wave (See Bredmose et al. (2015); Ma et al. (2016); Hu et al. (2017); Chuang et al. (2017)). Indeed, the breaking wave forces yield

25 the highest hydrodynamic loads on substructures in shallow water, particularly plunging breaking waves. For example, the estimation of the wave impact force (load and pressure) is important in order to design offshore wind turbines substructures. Concerning liquid jet injection, linear stability analysis has shown 
that the gas Mach number has a dramatic impact on the growing rate of the instability and the wavelength of the most unstable wave (Zhou and Lin, 1992 , Funada et al., 2006, Tharakan and Ramamurthi, 2010). This demonstrates the crucial importance of considering the compressibility effects.

The use of a compressible, variable-density solver is also mandatory to describe phase change in complex configurations such as liquid jet injection or breaking waves. Handling phase change in numerous gas inclusion, each possessing their own gas density evolution and thermodynamic pressure, is not straightforward. In our previous works, the phase change description was based on an incompressible solver : first by using a passive scalar to focus on the mixing process (Duret et al., 2012), then by computing the evaporation rate based 40 on temperature and species equations, allowing to evaluate the velocity jump condition and gas dilatation (Tanguy et al., 2007, Duret et al., 2014). The latter formulation is encouraging but is not suitable with the presence of multiple gas inclusions or in a confined environment, since the gas and vapour density remains constant in that formalism.

The main objective of this article is to break a scientific barrier concerning the numerical simulation of compressible liquid-gas flows. Indeed, the majority of accurate numerical methods used in two-phase flows simulation with interface tracking/capturing method can be categorized in two families: incompressible (for instance Ménard et al. (2007)) or compressible method (Kuila et al., 2015).

so Very different formalism are used to solve the governing equations (Poisson solver versus Riemann solver) and only few collaborations exist between the "incompressible" and the "compressible" communities. The drawback of these methods is their inability to capture accurately and smoothly two-phase flows with a wide evolution of the compressibility effects, from (very) low Mach num55 ber to high Mach number.

This issue was solved in the case of single phase flows or reacting flows with the low Mach approach (reviewed in Schochet (2005)), which has been used to represent the expansion/compression of gas and density variations. However, this kind of approach is not straightforward in the context of two-phase flows. 
60 The existence of inclusions (bubbles) of gas inside a liquid imposes a complex way to take into account the gas density variation inside each inclusion. Indeed, the thermodynamic pressure difference in each inclusion make the equation difficult to solve with a one-field approach (See Daru et al. (2010)). A solution is to account for the density variation by integrating the incoming fluxes on the

65 boundary of the bubble, or use an extension to take into account the jump of thermodynamics pressure. Nevertheless, gas inclusions and their surface have to be tracked to properly estimate the mean density variation. It means that it will be impossible to simulate realistic large scale configurations with numerous gas inclusions because of the computational cost involved.

70 Moreover, most numerical methods in the literature does not take into account all physical phenomena that can be encountered in two-phase flows: compressibility, interface deformation/breakup, viscosity, surface tension and discontinuities at the interface. For example, the recent $2 \mathrm{D}$ study of Bredmose et al. (2015) admits the need to smear out the liquid-gas interface, which has a negative impact on the wave propagation from air to water, and also modeled the compressible gas by considering an homogeneous mixture of incompressible liquid and ideal gas as a first approach. Another example is the diffuse interface method (Saurel and Abgrall (1999)) based on the phase field model. It consists in the resolution of a unique set of equations for all the phases of the flow. The so phases are represented by a function that tends toward 0 in the gas part for example and 1 in the liquid part. This function, which follows a Cahn-Hilliard equation, varies continuously around the interface creating an artificial mixing zone of both phases. In order to have the right jump conditions across the interface, relaxation equations are solved and the surface tension is taken into

85 account by modifying the stress tensor. Some works include surface tension in compressible multiphase flows like Perigaud and Saurel (2005) or Braconnier and Nkonga 2009). This kind of interface is often used in parallel of a Riemann solver for high Mach number flows (Petitpas et al. (2009) Chiapolino et al. (2017)) whose numerical diffusion stabilize the interface. However, the

9o lack of interface sharpness is an issue in these methods to apply proper jump 
conditions or to evaluate accurately the interface curvature.

In regards to atomization processes, most DNS/LES studies are considered incompressible, despite the fact that the initial injection velocity can be close or higher than the celerity of sound in the gas phase. For instance, in the ${ }_{95}$ Engine Combustion network injector called "Spray A" (Knudsen et al. (2017)) the injection velocity is around $600 \mathrm{~m} . \mathrm{s}^{-1}$. Pressures waves developing in front of the liquid jet are then likely to be observed. Another important topic is the cavitation process inside injectors nozzles, taking into account compressibility will give a new insight into identifying cavitation zones in the liquid phase and understanding the effect of cavitation on the atomization process. This illustrates the need of new and original numerical developments to fully resolve both phases and also acoustic effects.

Some attempts has been made to solve these specifics issues. The early pioneering work of Harlow and Amsden (1971) paved the way for the so-called "pressure-based" methods. In their work, they used an ICE (Implicit Continuous fluid Eulerian) method to solve all flow speeds in single-phase flows. They combined both explicit resolution for high flow speeds and implicit resolution for low flow speeds, by using weighted constants which depend on sound speed. More recently, these kind of methods has been extended to two-phase flows applications. Miller et al. (2013) proposed one of the first pressure-based formalism using a Volume Of Fluid (VOF) interface capturing method in OpenFOAM, showing encouraging results on underwater explosions and shock tubes configurations with large density ratio. However, the author admits that mass is not well conserved and jump conditions for variable such as density, viscosity are smeared out over a few cells. Huber et al. (2015) used a primitive formulation of the compressible Navier-Stokes equations to consider compressible two-phase flows, combined with a pressure-based method similar to the one developed in single phase flow context by Kwatra et al. (2009). Acoustics terms where treated implicitly along with a proper description of surface tension forces. This method has been compared with a standard compressible solver (preconditioned explicit HLLC), showing that better results has been obtained with the pressure-based 
method on a oscillating bubble configuration (Rayleigh-Plesset theory). Boger et al. (2014) performed a similar study by using a pressure-based method to study shock-droplet interaction. However, these methods are coupled with a Level-Set approach, making turbulent atomization difficult to handle (mass loss) and viscous effects are not taken into account.

This work aims at providing numerical tools, allowing simulations of twophase flows covering a large range of Mach number, incorporating surface tension, acoustic/compressible effects, large density ratio, proper jump conditions, viscous effects, at High Reynolds and High Weber number. We propose achieving this challenging task by improving the two-phase flows aspect of the pressurebased method proposed by Huber et al. (2015) and Miller et al. (2013) by combining it with an accurate and conservative interface representation : the Coupled Level Set/VOF interface capturing method. The main emphasis of this study is on the computation of Low Mach number configurations but the method can be used as well in shock waves configurations.

In the following part of this work, the constitutive equations and numerical procedures are first described without phase change. Next, validations cases such as a gas-water shock tube and an oscillating water column configuration illustrates the accuracy and robustness of the method. Finally, the potential of the method is shown by studying the impact of compressibility and dilatation induced by a vaporization source term in the pressure and VOF equation in the two-phase flow Homogeneous Isotropic Turbulence (HIT) configuration.

\section{Governing Equations}

The joint LS/VOF method is coupled with a projection method to carry out the Direct Numerical Simulations of compressible Navier-Stokes equations :

$$
\left\{\begin{array}{l}
\frac{\partial \rho}{\partial t}+\vec{\nabla} \cdot(\rho \vec{u})=0 \\
\frac{\partial \rho \vec{u}}{\partial t}+\vec{\nabla} \cdot(\rho \vec{u} \otimes \vec{u})=-\vec{\nabla} P+\vec{\nabla} \cdot\left(2 \mu \epsilon+\left(\zeta-\frac{2}{3} \mu\right) \vec{\nabla} \cdot \vec{u} \bar{I}\right)+\rho \vec{f}
\end{array}\right.
$$

with $\rho$, the density ; $\vec{u}$, the velocity ; $P$, the pressure ; $\mu$, the dynamic viscosity ; $\epsilon=\frac{1}{2}\left(\vec{\nabla} \vec{u}+\vec{\nabla} \vec{u}^{t}\right)$, the strain rate tensor ; $\zeta$, the second viscosity ; 
$\vec{f}$, the force of volume by unit of mass and $\bar{I}$, the identity matrix. Here, both liquid and gas are considered as compressible. On the interface, surface tension is taken into account by considering the variables jump across the liquid/gas interface :

$$
[\vec{n} \cdot(P \bar{I}-\bar{\tau}) \cdot \vec{n}]=\sigma \kappa
$$

with $\sigma$, the surface tension ; $\kappa$, the total curvature $; \vec{n}$, the normal to the interface ; $\bar{\tau}$, the viscous strain tensor defined by $\bar{\tau}=2 \mu \epsilon+\left(\zeta-\frac{2}{3} \mu\right) \vec{\nabla} \cdot \vec{u} \bar{I}$ and the convention $[A]=A_{l}-A_{g}$ for the variables jump across the interface.

Eq 1 is closed by two equations of state (EOS) for each phase. For the liquid, a Tait equation has been used. Concerning the gas phase, an ideal gas and an isentropic process have been considered (Laplace's law) :

$$
\left\{\begin{array}{l}
\rho_{g}=\left(\frac{P}{C_{\gamma}}\right)^{\frac{1}{\gamma_{g}}} \\
\rho_{l}=\rho_{0}\left(\frac{P-P_{0}}{B}+1\right)^{\frac{1}{\gamma_{l}}}
\end{array}\right.
$$

The $g$ and $l$ indexes denote the gas and the liquid, respectively $; \gamma$ represents the adiabatic index $; P_{0}$, a reference pressure $; \rho_{0}$, a reference density $; C_{\gamma}$, a constant depending on the initial condition of the ideal gas studied and $B$, a constant depending on the bulk modulus of the considered fluid.

\section{Numerical Methods}

\subsection{Interface capturing method}

The interface is solved using a CLSVOF algorithm. This method allows an accurate representation of the interface with the Level Set function and the mass conservation with the VOF method. The general algorithm can be found in Ménard et al. (2007). However, in this work an additional term due to compressibility has to be taken into account in the liquid volume fraction equation. The new formulation of this equation is :

$$
\frac{\partial \alpha_{l}}{\partial t}+\vec{\nabla} \cdot\left(\alpha_{l} \vec{u}\right)=\alpha_{l}\left(1-\alpha_{l}\right) D+\alpha_{l} \vec{\nabla} \cdot \vec{u}
$$


with $\alpha_{l}$, the liquid volume fraction and $D$, a term representing the fluid compressibility and defined by

$$
D=\frac{1}{\rho_{g}} \frac{D \rho_{g}}{D t}-\frac{1}{\rho_{l}} \frac{D \rho_{l}}{D t}
$$

where $\frac{D}{D t}$ is the material derivative. Eq 4 is derived from the continuity equation of Eq 1 by considering $\rho=\alpha_{l} \rho_{l}+\alpha_{g} \rho_{g}$ and $\alpha_{g}=1-\alpha_{l}$. Contrary to most studies using the CLSVOF framework, here the pressure $P$ and the densities $\rho_{l}$ and $\rho_{g}$ are local variables. In the incompressible CLSVOF algorithm, the liquid volume fraction and Level Set transport equations have the same formulation and are split in the three space directions. For the sake of clarity, only the VOF transport equation is shown below :

$$
\left\{\begin{array}{l}
\frac{\tilde{\alpha_{l}}-\alpha_{l}^{n}}{\Delta t}+\frac{\left(\alpha_{l}^{n} u\right)_{i+\frac{1}{2}}-\left(\alpha_{l}^{n} u\right)_{i-\frac{1}{2}}}{\Delta x}=\tilde{\alpha}_{l} \frac{u_{i+\frac{1}{2}}-u_{i-\frac{1}{2}}}{\Delta x} \\
\frac{\hat{\alpha}_{l}-\tilde{\alpha_{l}}}{\Delta t}+\frac{\left(\tilde{\alpha}_{l} v\right)_{j+\frac{1}{2}}-\left(\tilde{\alpha}_{l} v\right)_{j-\frac{1}{2}}}{\Delta y}=\hat{\alpha}_{l} \frac{v_{j+\frac{1}{2}}-v_{j-\frac{1}{2}}}{\Delta y} \\
\frac{\tilde{\alpha}_{l}-\hat{\alpha_{l}}}{\Delta t}+\frac{\left(\hat{\alpha_{l}} w\right)_{k+\frac{1}{2}}-\left(\hat{\alpha_{l}} w\right)_{k-\frac{1}{2}}}{\Delta z}=\bar{\alpha}_{l} \frac{w_{k+\frac{1}{2}}-w_{k-\frac{1}{2}}}{\Delta z}
\end{array}\right.
$$

Then, a final equation couples the three directions. To be coherent with the Level Set equation that has no additional term in compressible formulation, the compressibility term $D$ of $\mathrm{Eq} 4$ is only added in the final equation and $\mathrm{Eq} 6$ is solved following the incompressible formalism of Ménard et al. (2007). The $\alpha_{l}\left(1-\alpha_{l}\right) D$ term in Eq 4 is implicited in two ways according to the sign of $D$ :

$$
\begin{aligned}
& \frac{\alpha_{l}^{n+1}-\bar{\alpha}_{l}}{\Delta t}=-\bar{\alpha}_{l} \frac{w_{k+\frac{1}{2}}-w_{k-\frac{1}{2}}}{\Delta z}-\hat{\alpha}_{l} \frac{v_{j+\frac{1}{2}}-v_{j-\frac{1}{2}}}{\Delta y}-\tilde{\alpha}_{l} \frac{u_{i+\frac{1}{2}}-u_{i-\frac{1}{2}}}{\Delta x} \\
& +\alpha_{l}^{n+1}\left(\frac{w_{k+\frac{1}{2}}-w_{k-\frac{1}{2}}}{\Delta z}+\frac{v_{j+\frac{1}{2}}-v_{j-\frac{1}{2}}}{\Delta y}+\frac{u_{i+\frac{1}{2}}-u_{i-\frac{1}{2}}}{\Delta x}\right)+A_{\text {comp }} \\
& \text { with }\left\{\begin{array}{lllll}
A_{\text {comp }}=\alpha_{l}^{n+1}\left(1-\bar{\alpha}_{l}\right) D & \text { if } & D<0 & \text { and } & \alpha_{l}<1 \\
A_{\text {comp }}=\bar{\alpha}_{l}\left(1-\alpha_{l}^{n+1}\right) D & \text { if } & D>0 & \text { and } & \alpha_{l}>0
\end{array}\right.
\end{aligned}
$$

A reinitialization step of the Level Set function is performed at each time step, similar to previous works done in the incompressible framework (Tanguy 
et al. 2007; Ménard et al., 2007). All the geometrical informations of the interface are obtained with the Level Set function $\phi$. The curvature $\kappa$ is calculated by $\kappa=\vec{\nabla} \cdot \vec{n}$ with $\vec{n}=\vec{\nabla} \phi /|\vec{\nabla} \phi|$, the normal to the interface.

\subsection{Projection method}

Then, to compute velocity and pressure, the momentum equation of Eq1 is solved using a projection method adapted to compressible formulation. This method allows to decouple velocity and pressure. An intermediate velocity is first calculated without the pressure term $-\vec{\nabla} P$ and the surface tension term :

$$
\vec{u}^{*}=\underbrace{\frac{\rho^{n} \vec{u}^{n}}{\rho^{*}}-\Delta t \frac{\vec{\nabla} \cdot\left((\rho \vec{u})^{n} \otimes \vec{u}^{n}\right)}{\rho^{*}}}_{\mathrm{I}}+\underbrace{\Delta t \frac{\vec{\nabla} \cdot\left(2 \mu \epsilon-\frac{2}{3} \mu \vec{\nabla} \cdot \vec{u}^{n} \bar{I}\right)}{\rho^{*}}}_{\mathrm{II}}+\Delta t \vec{f}
$$

$\rho^{*}$ corresponds to the density calculated with $\rho=\alpha_{l} \rho_{l}+\alpha_{g} \rho_{g}$ but with the new value of $\alpha_{l}$ obtained after the interface resolution. The viscous term $I I$ is solved accordingly with the method presented in Sussman et al. (2007). This method takes into account directly the viscous tensor jump across the interface. It was originally developed in a incompressible formalism, so the term $\left(-\frac{2}{3} \mu \vec{\nabla} \cdot \vec{u} \bar{I}\right)$ was added in this formulation. The second viscosity $\zeta$ is neglected in this work. Moreover, no artificial viscosity has been added in this work.

Regarding the convection term $I$, the method of Vaudor et al. (2017) has been used ensuring consistent mass and momentum fluxes computation. The mesh is an eulerian staggered grid so the velocity is computed on the faces of the cells and the other variables (pressure, density, liquid volume fraction, ...) are computed in the center of the cells. This method initially proposed by Rudman (1998) consists in calculating the mass flux $\rho \vec{u}$ in the center of the cell by using the continuity equation. The mass flux has to be known in the center of the cell in order to have a second order centered scheme for the divergence operator. A detailed explanation of the algorithm can be found in Vaudor et al. (2017).

Then, the momentum equation is discretized in the following way by using 
the intermediate velocity obtained previously.

$$
\vec{u}^{n+1}=\vec{u}^{*}-\Delta t \frac{\vec{\nabla} P}{\rho^{*}}
$$

By applying the divergence operator to Eq, 9 , an Helmholtz equation for the pressible solvers, the divergence of the velocity is no longer zero. Further details regarding the development of the pressure equation are available in Appendix A.

$$
\begin{aligned}
& -\vec{\nabla} \cdot\left(\frac{\vec{\nabla} P^{n+1}}{\rho^{*}}\right)+\left(\frac{\alpha_{l}}{\rho_{l} c_{l}^{2} \Delta t^{2}}+\frac{\alpha_{g}}{\rho_{g} c_{g}^{2} \Delta t^{2}}\right) P^{n+1}= \\
& \left(\frac{\alpha_{l}}{\rho_{l} c_{l}^{2} \Delta t^{2}}+\frac{\alpha_{g}}{\rho_{g} c_{g}^{2} \Delta t^{2}}\right)\left(P^{n}-\Delta t \vec{u} . \vec{\nabla} P^{n}\right)-\frac{\vec{\nabla} \cdot \vec{u}^{*}}{\Delta t}
\end{aligned}
$$

with $c_{l}$ and $c_{g}$, respectively the sound speed for liquid and gas. The $\vec{\nabla} \cdot \vec{u}^{*}$ 215 term is solved with a second order centered scheme and $\vec{u} . \vec{\nabla} P$, with a fifth order WENO scheme (Shu, 1997). A Ghost Fluid Method (GFM) is used to apply the pressure jump due to surface tension forces (Ménard et al., 2007). The advantage of the GFM is a more realistic representation of the interface (sharp, infinitely thin) : jump conditions are directly added at the interface position through a local modification of the numerical scheme. The distance of the interface is provided by the Level Set function. The final velocity is computed by Eq.9 using a second order centered scheme for the pressure gradient. A second order predictor-corrector Runge Kutta scheme have been used for temporal integration. Finally, the density and sound speed for liquid and gas and the total density are updated with the EOS Eq 3 . The compressibility term $D$ used in $\mathrm{Eq} 4$ is solved to be coherent with the pressure term :

$$
D^{n+1}=\left(\frac{1}{\rho_{g} c_{g}^{2}}-\frac{1}{\rho_{l} c_{l}^{2}}\right)\left(\frac{P^{n+1}-P^{n}}{\Delta t}+\vec{u} . \vec{\nabla} P\right)
$$

Concerning the time step calculation, a CFL condition similar to the one used by Kang et al. (2000) is used. Thanks to the implicit resolution of the 
acoustics terms in the pressure equation, the CFL condition is the same used than the one obtained with an acoustic CFL based on the sound speed. For $C F L=1$, the CFL condition can be written as :

$$
\Delta t\left(\frac{\left(C_{c f l}+V_{c f l}\right)+\sqrt{\left(C_{c f l}+V_{c f l}\right)^{2}+4\left(G_{c f l}\right)^{2}+4\left(S_{c f l}\right)^{2}}}{2}\right) \leq 1
$$

with $C_{c f l}=\frac{|u|_{\max }}{\Delta x}+\frac{|v|_{\max }}{\Delta y}+\frac{|w|_{\max }}{\Delta z}, V_{c f l}=\left(\frac{2}{\Delta x^{2}}+\frac{2}{\Delta y^{2}}+\frac{2}{\Delta z^{2}}\right) * \max \left\{\frac{\mu_{l}}{\rho_{l}}, \frac{\mu_{g}}{\rho_{g}}\right\}$, $G_{c f l}=\sqrt{\frac{|g|}{\Delta y}}$ and $S_{c f l}=\sqrt{\frac{\sigma|\kappa|}{\min \left\{\rho_{g}, \rho_{l}\right\} *(\min \{\Delta x, \Delta y, \Delta z\})^{2}}}$.

\section{Results}

\subsection{Gas-water shock tube}

This validation case is an underwater explosion configuration, based on the case III-A shown in $\mathrm{Hu}$ and Khoo (2004). Bubbles are generated by the explosion, and then expands quickly into water. A shock wave develops into the water and a rarefaction wave is reflected back into the explosive bubble. This case presents high density and pressure ratio, which are difficult to handle without robust numerical methods. The initial conditions are the following :

$$
(\rho, u, p, \gamma)= \begin{cases}(0.01,0,1000,2), & \text { if } x<0.5 \\ (1,0,1,7.15) & \text { if } x>0.5\end{cases}
$$

Figure 11 compare our results with the analytical solution, showing a good agreement with the theory. The wave speed is correctly captured as well as the density profile. The pressure profile is also well captured (Figure 2) without oscillations. This results demonstrate the accuracy and robustness of our pressure-based method in a two-phase flows context.

\subsection{Oscillating water column configuration}

In order to validate the method, an oscillating water column test case has 

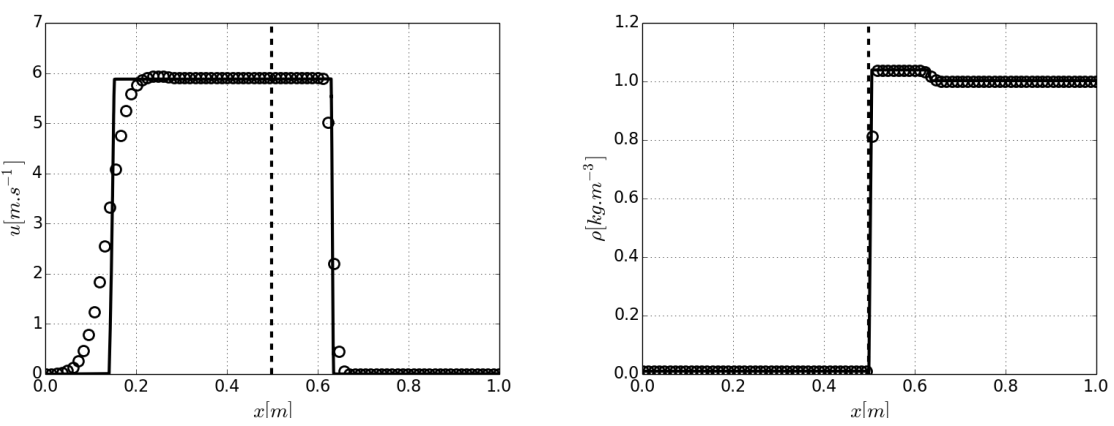

Figure 1: Velocity (left) and density (right) profiles at $t=8 \times 10^{-4} \mathrm{~s}$. The solid line represents the theory and the circles represent the numerical results for a 256 cells mesh and $d t=$ $8 \times 10^{-6} \mathrm{~s}$. The dashed line represents the liquid-gas interface location

between two air columns due to an initial pressure gradient. The liquid compresses a first air column and then, when the pressure in this column exceeds the liquid inertia, the air pushes the water column toward the second air column and so on. This test case can be seen as a Rayleigh-Plesset test case, representing the oscillation of an air bubble inside liquid, but in a 1D cartesian geometry. This test case has been studied previously in Daru et al. (2010); Kadioglu et al. (2005); Koren et al. (2002) by imposing an initial velocity to the liquid column instead of a pressure gradient. However, no reference solution exists to compare our numerical results although a theoretical solution can be derived by using mass and momentum conservation and by considering the liquid incompressible. The mass conservation expressed with the flow rate conservation gives :

$$
\rho_{l} S u=\rho_{l} S \frac{d R}{d t}
$$

with $S$, a plane surface at a given position $x ; R$, the first gas-liquid interface position ; $\rho_{l}$ the liquid density and $u$, the velocity of the liquid in $x$ direction. By considering the liquid incompressible, we have :

$$
u=\frac{d R}{d t}
$$

So the velocity in the liquid is only a function of time. Then, the momentum 


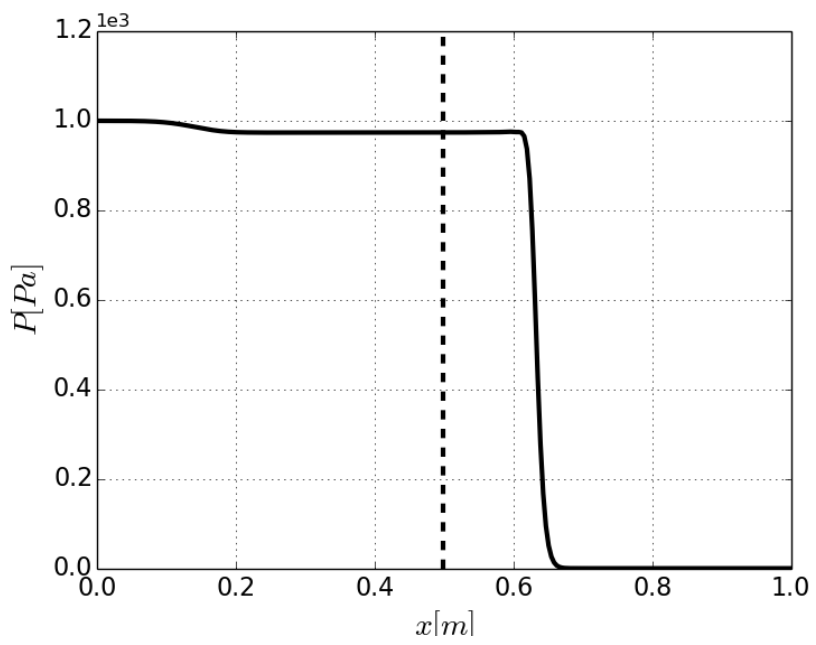

Figure 2: Pressure profiles at $t=8 \times 10^{-4} \mathrm{~s}$. The solid line represents the numerical result for a 256 cells mesh and $d t=8 \times 10^{-6} \mathrm{~s}$. The dashed line represents the liquid-gas interface location

equation in 1D without surface tension and viscosity, can be written as follow :

$$
\frac{\partial u}{\partial t}+u \frac{\partial u}{\partial x}=-\frac{1}{\rho_{l}} \frac{\partial P}{\partial x}
$$

By injecting Eq14 in Eq15, we obtain :

$$
\frac{d^{2} R}{d t^{2}}=-\frac{1}{\rho_{l}} \frac{\partial P}{\partial x}
$$

Finally, by integrating Eq 16 between the position of the first interface gasliquid $R_{1}$ and the position of the second interface $R_{2}$, the equation for the evolution of the first interface position in function of time is obtained :

$$
\frac{d^{2} R}{d t^{2}}=\frac{\left.P\right|_{R_{1}}-\left.P\right|_{R_{2}}}{\rho_{l} L}
$$

with $L=R_{2}-R_{1}=c s t$, the liquid length. The pressures in $R_{1}$ and $R_{2}$ are obtained with an equation of state. Then, the velocity is calculated with Eq.14. No analytical solution was found but a reference solution can be easily computed. It is worth mentioning that the reference solution is considering no 

numerical method presented here. Consequently, slight differences should be observed on the results when comparing both approaches.

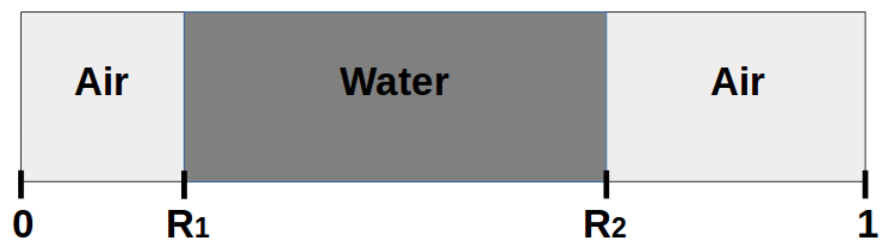

Figure 3: Oscillating Water Column test case

The initial values for density and pressure are referenced in Table 1 . The pressure in the first air column is twice the pressure in the second air column and it follows a linear profile in the water column. The water column is located between $R_{1}=0.1 \mathrm{~m}$ and $R_{2}=0.8 \mathrm{~m}$ and the total domain measures $1 \mathrm{~m}$. For the Tait equation in Eq.3, the parameters are $B=3.31 \times 10^{8} \mathrm{~Pa}, \mathrm{P}_{0}=10^{5} \mathrm{~Pa}$ and $\rho_{0}=1000 \mathrm{~kg} \cdot \mathrm{m}^{-3}$.

\begin{tabular}{|c|c|c|}
\hline Air & Water & Air \\
$0 \leq x \leq R_{1}$ & $R_{1} \leq x \leq R_{2}$ & $R_{2} \leq x \leq 1$ \\
\hline$\gamma_{g}=1.4$ & $\gamma_{l}=7$ & $\gamma_{g}=1.4$ \\
$\rho_{L}=1.169 \mathrm{~kg} \cdot \mathrm{m}^{-3}$ & $\rho=1000 \mathrm{~kg} \cdot \mathrm{m}^{-3}$ & $\rho_{R}=0.5845 \mathrm{~kg} \cdot \mathrm{m}^{-3}$ \\
$P_{L}=10^{5} \mathrm{~Pa}$ & $P=\frac{P_{R}-P_{L}}{R_{2}-R_{1}} x+\frac{P_{L} R_{2}-P_{R} R_{1}}{R_{2}-R_{1}}$ & $P_{R}=5 \times 10^{4} \mathrm{~Pa}$ \\
\hline
\end{tabular}

Table 1: Initial conditions for the oscillating water column test case. The $L$ and $R$ indexes mean respectively left and right.

For this configuration, the Mach number reaches a maximum value of $M_{a}=$ $4.45 \times 10^{-3}$. All the boundary conditions are considered as symmetric.

Figure 4 compares the evolution in function of time of the velocity of the liquid for different mesh size to the incompressible reference solution. As expected, the velocity oscillates ; initially, the liquid accelerates in the positive $\mathrm{x}$ 
here ; however, this $C F L$ condition is based on the convective speed (similar to Kwatra et al. (2009) and Huber et al. (2015)). The corresponding time step is about 100 times greater than the one obtained from the $C F L$ condition based on the sound speed.

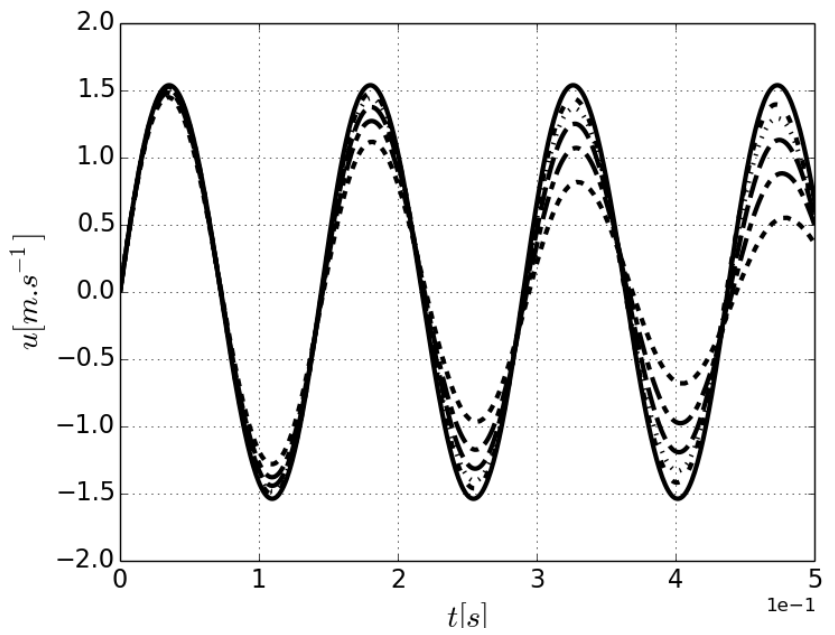

Figure 4: Evolution of the velocity in function of time for different meshes, CFL $=0.1 .-32$ cells,--- 64 cells, ---128 cells, $\cdots 256$ cells, - - 512 cells. The solid line is the reference solution

Regarding the mass conservation, a better accuracy is obtained by refining the mesh (Figure 5) and the relative error is less than $0.1 \%$ for meshes with more than 256 cells, as shown in Figures 5 and 6 .

By using a $C F L=0.01$ condition, namely a time step about 10 times greater than the one obtained with the acoustic $C F L$, a convergence is also observed 


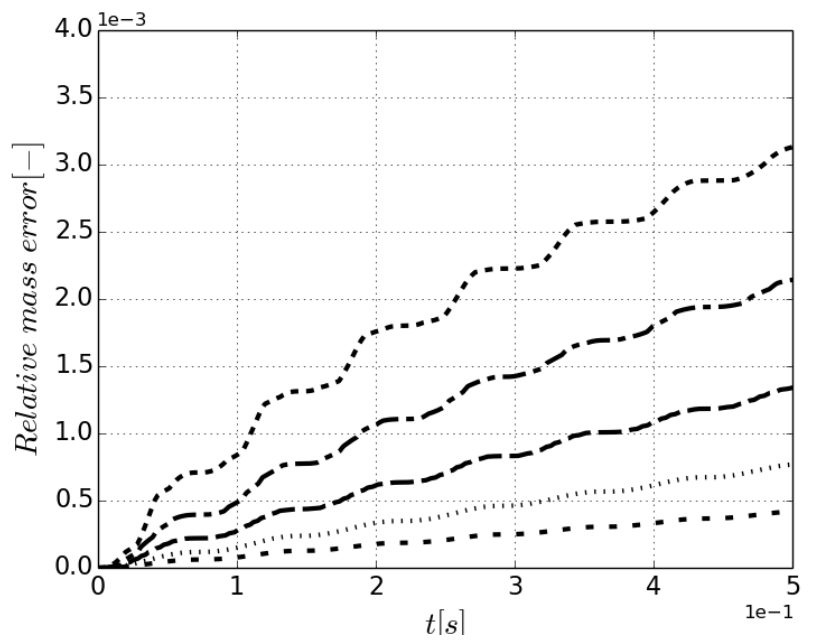

Figure 5: Relative mass of liquid error in function of time for different meshes, $\mathrm{CFL}=0.1 .-$ 32 cells, ---64 cells, ---128 cells, $\cdots 256$ cells, - - - 512 cells

the curve is closer to the reference solution but with a slight delay in terms of frequency. Concerning the mass conservation, an error about 10 times lower is noticed for both liquid and air.

The relative errors for the different meshes are shown in Table 2 for the first maximum for the velocity and for the frequency calculated between the two first maximum of velocity. In all cases, the relative error decreases by refining the mesh, illustrating the method accuracy and convergence.

\begin{tabular}{|c|c|c|c|c|c|}
\hline$N_{x}$ & 32 & 64 & 128 & 256 & 512 \\
\hline Amplitude Error & 5.82 & 3.66 & 2.19 & 1.30 & 0.49 \\
\hline Frequency Error & 1.49 & 0.87 & 0.65 & 0.43 & 0.35 \\
\hline
\end{tabular}

Table 2: Relative error (\%) for a $\mathrm{CFL}=0.1, N_{x}$ is the number of cells. 


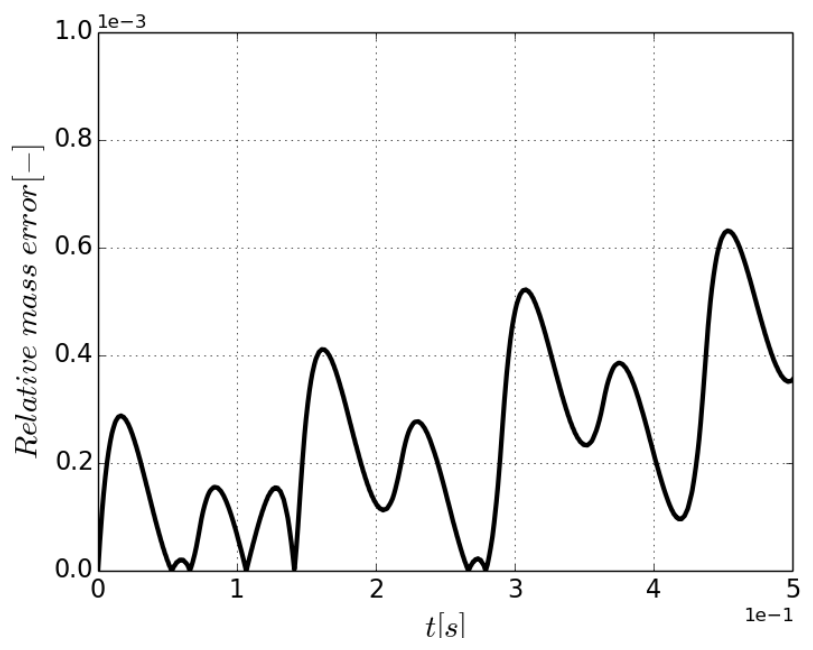

Figure 6: Relative mass of air error in function of time for 512 cells, $\mathrm{CFL}=0.1$

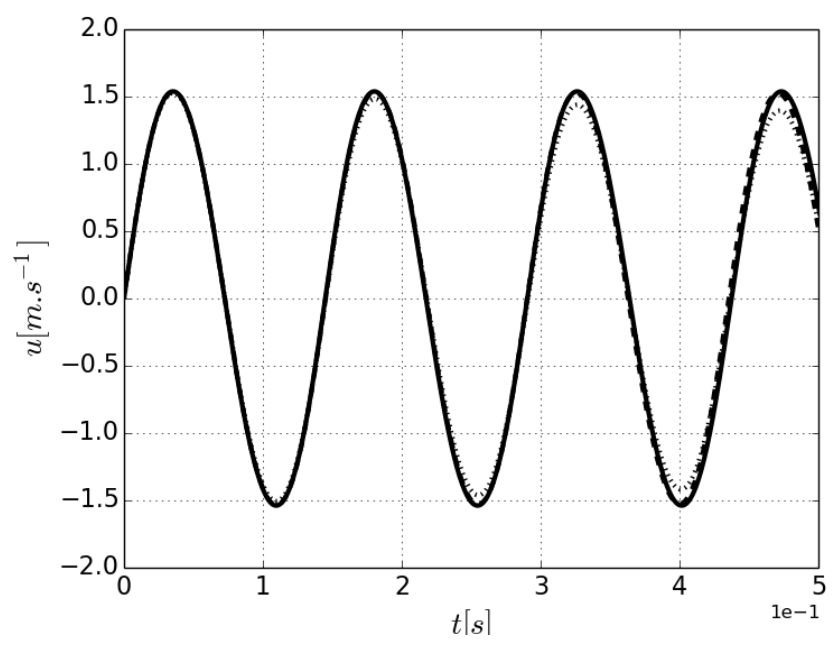

Figure 7: Evolution of the velocity in function of time for 512 cells.- - $\mathrm{CFL}=0.01, \cdots \mathrm{CFL}=0.1$.

The solid line is the reference solution 

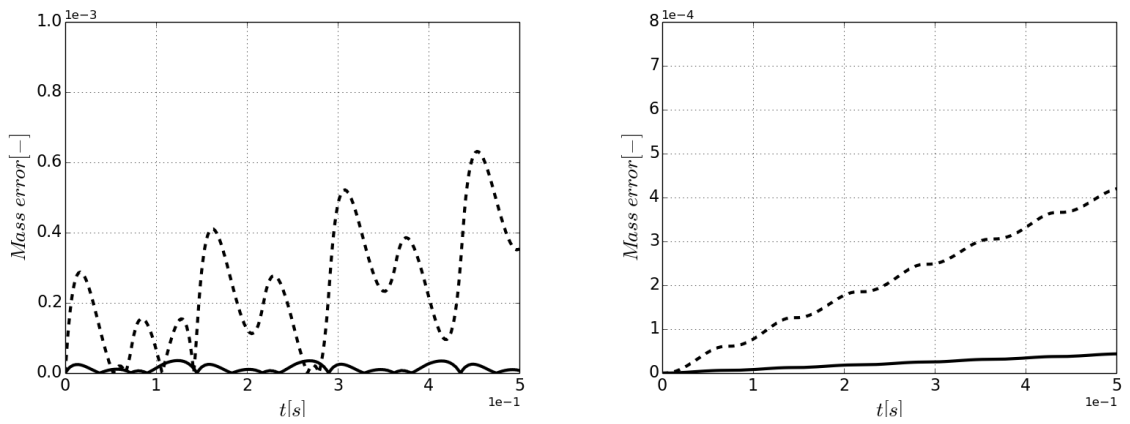

Figure 8: Relative mass error of liquid (right) and gas (left) in function of time for 512 cells.

Dashed line represents $\mathrm{CFL}=0.1$ and solid line represents $\mathrm{CFL}=0.01$ 


\subsection{HIT configuration with phase change}

Similarly to Duret et al. (2012), the idea is to investigate the influence of the vaporization process inside the two phase flows HIT, but this time the impact of vaporization on the flow dynamic (pressure, velocity) is considered. Contrary to the study of Duret et al. (2012) that used a passive scalar to represent the vaporization and mixing process, in this work a vaporization source term is directly introduced in the continuity and pressure equation. Consequently, the gas density will increase due to the vaporization process.

\subsubsection{Representation of the evaporation process}

In this configuration, phase change is considered. By splitting the continuity equation in Eq1 1 into a liquid density equation and a gas density equation

$325(\mathrm{Eq} 18)$, a mass source term is introduced :

$$
\left\{\begin{array}{l}
\frac{\partial \alpha_{l} \rho_{l}}{\partial t}+\vec{\nabla} \cdot\left(\alpha_{l} \rho_{l} \vec{u}\right)=\dot{m} \\
\frac{\partial \alpha_{g} \rho_{g}}{\partial t}+\vec{\nabla} \cdot\left(\alpha_{g} \rho_{g} \vec{u}\right)=-\dot{m}
\end{array}\right.
$$

with $\dot{m}$, the mass source term in $\left[\mathrm{kg} \cdot \mathrm{m}^{-3} \cdot \mathrm{s}^{-1}\right]$. It is negative when there is an evaporation of the liquid. Then, liquid and gas volume fraction equations are obtained by developing Eq,18.

$$
\left\{\begin{array}{l}
\frac{\partial \alpha_{l}}{\partial t}+\vec{\nabla} \cdot\left(\alpha_{l} \vec{u}\right)=-\frac{\alpha_{l}}{\rho_{l}} \frac{D \rho_{l}}{D t}+\frac{\dot{m}}{\rho_{l}} \\
\frac{\partial \alpha_{g}}{\partial t}+\vec{\nabla} \cdot\left(\alpha_{g} \vec{u}\right)=-\frac{\alpha_{g}}{\rho_{g}} \frac{D \rho_{g}}{D t}-\frac{\dot{m}}{\rho_{g}}
\end{array}\right.
$$

By adding these two equations, the divergence of velocity is obtained :

$$
\vec{\nabla} \cdot \vec{u}=-\frac{\alpha_{l}}{\rho_{l}} \frac{D \rho_{l}}{D t}-\frac{\alpha_{g}}{\rho_{g}} \frac{D \rho_{g}}{D t}+\dot{m}\left(\frac{1}{\rho_{l}}-\frac{1}{\rho_{g}}\right)
$$

330

Finally, by adding and deducting $\alpha_{l} \vec{\nabla} \cdot \vec{u}$ in the liquid volume fraction equation and replacing the different terms by their expression, the liquid volume fraction equation containing phase change is expressed as followed :

$$
\frac{\partial \alpha_{l}}{\partial t}+\vec{\nabla} \cdot\left(\alpha_{l} \vec{u}\right)=\alpha_{l}\left(1-\alpha_{l}\right) D+\alpha_{l} \vec{\nabla} \cdot \vec{u}-\alpha_{l} \dot{m}\left(\frac{1}{\rho_{l}}-\frac{1}{\rho_{g}}\right)+\frac{\dot{m}}{\rho_{l}}
$$


The pressure equation is also modified when phase change occurs :

$$
\begin{array}{r}
-\vec{\nabla} \cdot\left(\frac{\vec{\nabla} P^{n+1}}{\rho^{*}}\right)+\left(\frac{\alpha_{l}}{\rho_{l} c_{l}^{2} \Delta t^{2}}+\frac{\alpha_{g}}{\rho_{g} c_{g}^{2} \Delta t^{2}}\right) P^{n+1}= \\
\left(\frac{\alpha_{l}}{\rho_{l} c_{l}^{2} \Delta t^{2}}+\frac{\alpha_{g}}{\rho_{g} c_{g}^{2} \Delta t^{2}}\right)\left(P^{n}-\Delta t \vec{u} \cdot \vec{\nabla} P^{n}\right)-\frac{\vec{\nabla} \cdot \vec{u}^{*}}{\Delta t}+\frac{\dot{m}}{\Delta t}\left(\frac{1}{\rho_{l}}-\frac{1}{\rho_{g}}\right)
\end{array}
$$

The new term in the pressure equation (Eq 22 comes from the new formu-

335

\subsubsection{Numerical configuration}

For this Homogeneous Isotropic Turbulence (HIT) validation case, the forcing and the physical parameters used in previous work (Duret et al. 2012) are applied but with a $256^{3}$ mesh and an initial liquid volume fraction $\phi=10 \%$. sure is $P=9.06 \times 10^{6} \mathrm{~Pa}$ and for the Tait equation, the parameters used are $B=1.038 \times 10^{9} \mathrm{~Pa}, P_{0}=10^{5} \mathrm{~Pa}$ and $\rho_{0}=750 \mathrm{~kg} . \mathrm{m}^{-3}$. The adiabatic index for the liquid is $\gamma_{l}=1.215$ and for the gas is $\gamma_{g}=1.4$. For the evaporation, a constant vaporization rate $\dot{m}=-1 \mathrm{~kg} \cdot \mathrm{cm}^{-3} \cdot \mathrm{s}^{-1}$ is chosen. This value is only quently, the gas density is increased locally by the additional source term in the pressure equation, this can only be achieved by using a compressible or weakly-compressible formalism such as the one presented in this work. Moreover, the liquid-gas interface is directly influenced by the vaporization process through the VOF equation, meaning that the liquid mass diminishes, the gas mass increases and the total mass is conserved.

\subsubsection{Results}

Similarly to the incompressible HIT configuration presented in previous works, many breakup and coalescence events are observed (See Figure 9). Since the Mach number is very low in both phases, compressible effects are negligible : the gas density remains constant during the computation without evaporation. 


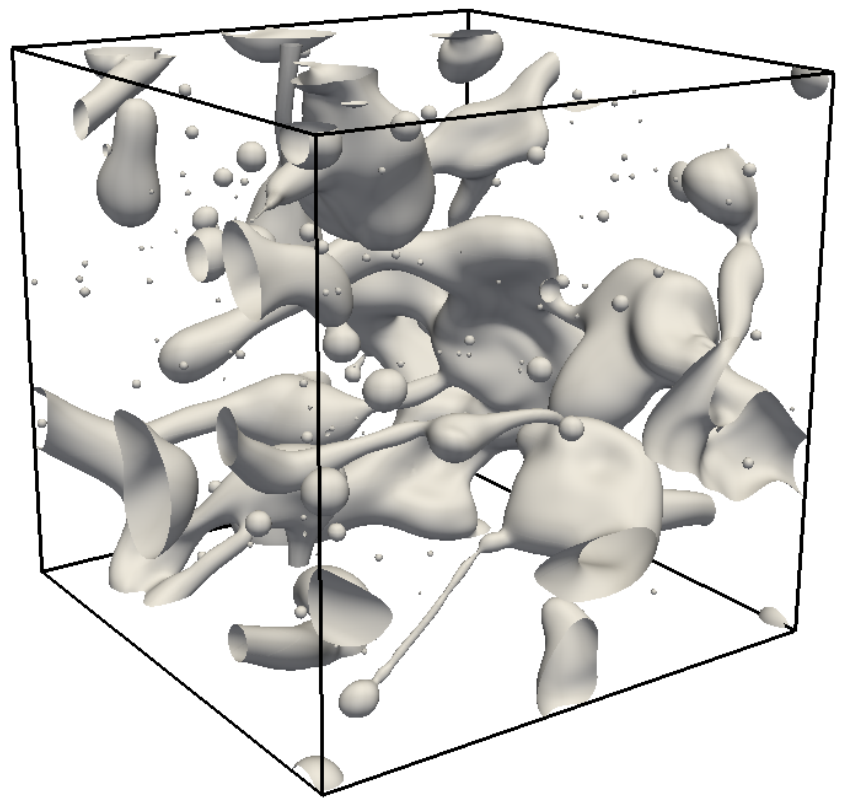

Figure 9: Surface representation of the HIT configuration with $\phi=10 \%$.

However, if phase change is considered, the gas density increases with time and a dilatation (Stefan flow) is generated close to the interface.

On Figure 10, the evolution of the mean gas density is represented. As expected, the density remains constant if no evaporation occurs and increases if the mass source term is not zero. Even if $\dot{m}$ is constant, the profile of the density is not linear because the surface quantity is not constant during the process. Consequently, the number of cells containing an interface varies. However, it is still possible to predict the gas density variation $\frac{\partial \rho_{g}}{\partial t}$ induced by vaporization if $\dot{m}$ is constant and evaporating cells are identified. Then the time evolution of $\rho_{g}$ is deduced, as shown on Figure 10 . The density profile obtained is very close to the reference one showing the capability of the algorithm to represent the evaporation process.

During evaporation, the total mass should be conserved ; the mass won by the gas should be lost by the liquid. This balance is observed on Figure 


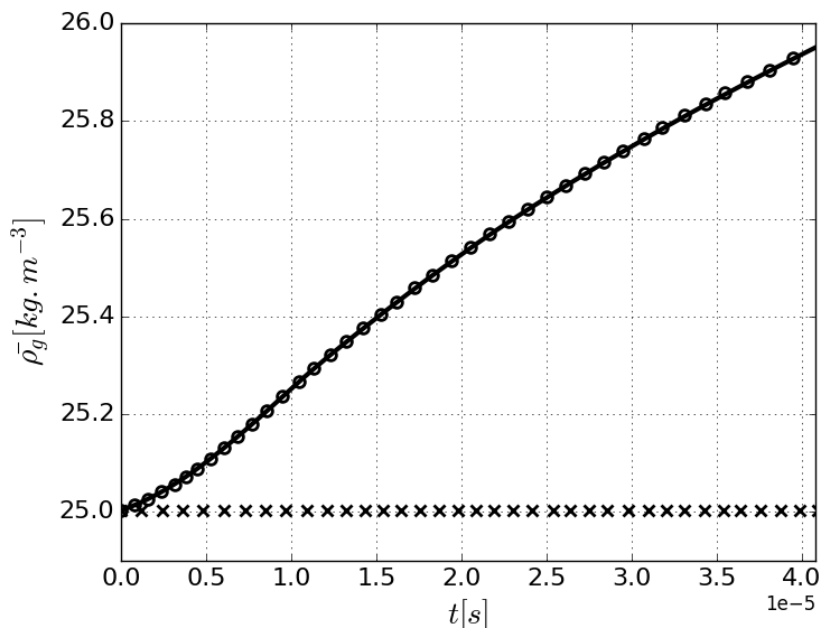

Figure 10: Evolution of the mean gas density in function of time. $\circ$ : results with evaporation, $\times$ : results without evaporation. Solid line : theoretical solution with evaporation.

11. where the mass of liquid is decreasing while the mass of gas is increasing with time. By adding these two masses, the total mass is estimated. The difference between the initial total mass and the final total mass obtained at the end of the computation is less than $0.2 \%$. This demonstrates the excellent mass conservation provided by the CLSVOF interface capturing method and the accurate computation of the mass transfer between liquid and gas phase due to vaporization.

\section{Conclusion}

A pressure based method is developed for low Mach number two-phase flows applications. The use of a projection method to decouple pressure and velocity allows a greater time step than the one imposed by the acoustic CFL condition. Indeed, the acoustic terms are solved implicitly in the pressure equation. This formalism takes into account the local variation of density in both phase due to compressibility and with (or without) phase change. 


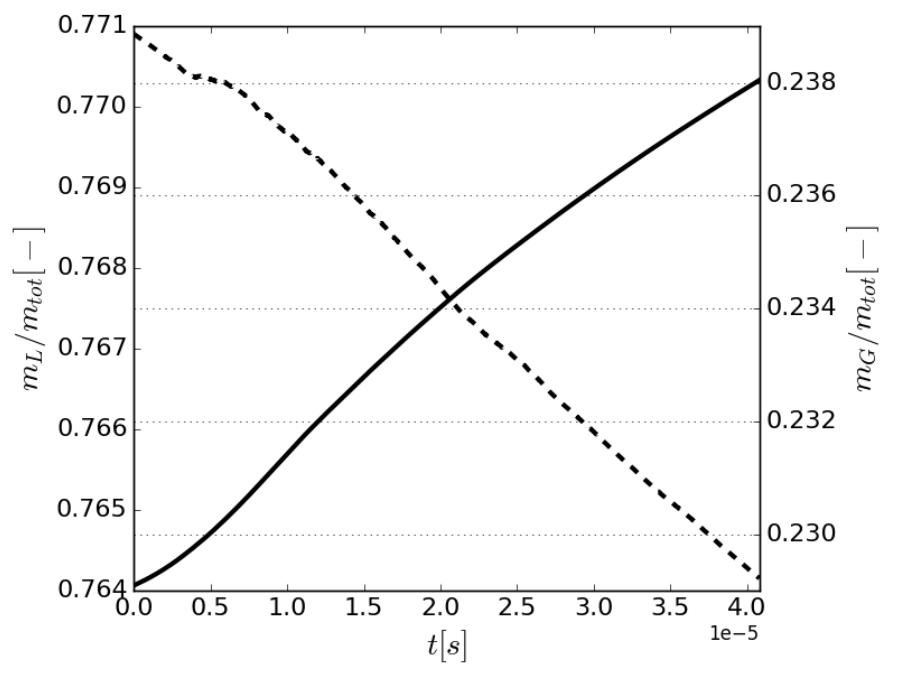

Figure 11: Evolution of the liquid mass $m_{l}$ and gas mass $m_{g}$ divided by the total mass $m_{t o t}$ in function of time. Dashed line (left axis) $: m_{l}(t)$, solid line (right axis) $: m_{g}(t)$.

ensure mass conservation and a sharp description of the interface. The standard incompressible VOF equation has been modified to take into account compressibility effects and the vaporization process.

The accuracy of the method is shown by comparing a reference solution with results obtained by this compressible DNS formulation in an oscillating water column configuration and a gas-water shock tube. Finally, the implementation of evaporation on a three-dimensional two-phase HIT configuration gives encouraging results that have to be pursued. An excellent mass conservation has been obtained by using this formalism, confirming the accuracy of the mass transfer estimation between liquid and gas phase due to vaporization. Moreover, this last configuration includes more complex phenomena like collisions and breakups, showing the robustness of this formalism in atomization context. Further works will be dedicated on coupling the vaporization rate to the species and energy equation in this compressible framework. 
Appendix A. Pressure equation

By applying the divergence operator on Eq, 9 , the following relation produced :

$$
\vec{\nabla} \cdot \vec{u}^{n+1}=\vec{\nabla} \cdot \vec{u}^{*}-\vec{\nabla} \cdot\left(\Delta t \frac{\vec{\nabla} P}{\rho^{*}}\right)
$$

First, $\vec{\nabla} \cdot \vec{u}^{n+1}$ is obtained by decomposing the density in the continuity equation into a liquid and gas density :

$$
\frac{\partial\left(\alpha_{l} \rho_{l}+\alpha_{g} \rho_{g}\right)}{\partial t}+\vec{\nabla} \cdot\left(\alpha_{l} \rho_{l} \vec{u}+\alpha_{g} \rho_{g} \vec{u}\right)=0
$$

405

Then, the liquid phase and the gas phase are separated in Eq A.2 and phase change is ignored in order to have :

$$
\left\{\begin{array}{c}
\frac{\partial \alpha_{l} \rho_{l}}{\partial t}+\vec{\nabla} \cdot\left(\alpha_{l} \rho_{l} \vec{u}\right)=0 \\
\frac{\partial \alpha_{g} \rho_{g}}{\partial t}+\vec{\nabla} \cdot\left(\alpha_{g} \rho_{g} \vec{u}\right)=0
\end{array}\right.
$$

By developing the derivatives and gathering the different terms :

$$
\left\{\begin{array}{c}
\frac{\partial \alpha_{l}}{\partial t}+\vec{\nabla} \cdot\left(\alpha_{l} \vec{u}\right)=-\frac{\alpha_{l}}{\rho_{l}} \frac{D \rho_{l}}{D t} \\
\frac{\partial \alpha_{g}}{\partial t}+\vec{\nabla} \cdot\left(\alpha_{g} \vec{u}\right)=-\frac{\alpha_{g}}{\rho_{g}} \frac{D \rho_{g}}{D t}
\end{array}\right.
$$

Then, by adding the two equations in EqA.4. the divergence of velocity is expressed as:

$$
\vec{\nabla} \cdot \vec{u}=-\frac{\alpha_{l}}{\rho_{l}} \frac{D \rho_{l}}{D t}-\frac{\alpha_{g}}{\rho_{g}} \frac{D \rho_{g}}{D t}
$$

410

By considering density as a function of pressure only, Eq A.5 can be written as follow :

$$
\vec{\nabla} \cdot \vec{u}=-\left(\frac{\alpha_{l}}{\rho_{l} c_{l}^{2}}+\frac{\alpha_{g}}{\rho_{g} c_{g}^{2}}\right) \frac{D P}{D t}
$$


with $c^{2}=\frac{\partial P}{\partial \rho}$. Finally, by injecting Eq A.6 in Eq A.1, developing the material derivative and dividing by $\Delta t$, an Helmholtz equation for pressure is obtained :

$$
\begin{array}{r}
-\vec{\nabla} \cdot\left(\frac{\vec{\nabla} P^{n+1}}{\rho}\right)+\left(\frac{\alpha_{l}}{\rho_{l} c_{l}^{2} \Delta t^{2}}+\frac{\alpha_{g}}{\rho_{g} c_{g}^{2} \Delta t^{2}}\right) P^{n+1} \\
=\left(\frac{\alpha_{l}}{\rho_{l} c_{l}^{2} \Delta t^{2}}+\frac{\alpha_{g}}{\rho_{g} c_{g}^{2} \Delta t^{2}}\right)\left(P^{n}-\Delta t \vec{u} \cdot \vec{\nabla} P^{n}\right)-\frac{\vec{\nabla} \cdot \vec{u}^{*}}{\Delta t}
\end{array}
$$

415

Boger, M., Jaegle, F., Weigand, B., Munz, C. D., Jun. 2014. A pressure-based treatment for the direct numerical simulation of compressible multi-phase flow using multiple pressure variables. Computers \& Fluids 96 (Supplement C), 338-349.

420

Braconnier, B., Nkonga, B., Sep. 2009. An all-speed relaxation scheme for interface flows with surface tension. Journal of Computational Physics 228 (16), $5722-5739$.

Bredmose, H., Bullock, G. N., Hogg, A. J., Feb. 2015. Violent breaking wave impacts. Part 3. Effects of scale and aeration. Journal of Fluid Mechanics 765,

425 82-113.

Chiapolino, A., Saurel, R., Nkonga, B., Jul. 2017. Sharpening diffuse interfaces with compressible fluids on unstructured meshes. Journal of Computational Physics 340, 389-417.

Chuang, W.-L., Chang, K.-A., Mercier, R., Jun. 2017. Impact pressure and void 430 fraction due to plunging breaking wave impact on a $2 \mathrm{~d}$ TLP structure. Exp Fluids 58 (6), 68.

Daru, V., Le Quéré, P., Duluc, M. C., Le Maître, O., Nov. 2010. A numerical method for the simulation of low Mach number liquid-gas flows. Journal of Computational Physics 229 (23), 8844-8867. 
Duret, B., Luret, G., Reveillon, J., Menard, T., Berlemont, A., Demoulin, F.X., 2012. DNS analysis of turbulent mixing in two-phase flows. International Journal of Multiphase Flow 40 (0), 93 - 105.

Duret, B., Qubeissi, M. A., Crua, C., Sahzin, S., 2014. Evaporating droplets: comparisons between DNS and modelling. In: 26th European Conference on $440 \quad$ Liquid Atomization and Spray Systems.

Funada, T., Joseph, D. D., Saitoh, M., Yamashita, S., Jan. 2006. Liquid jet in a high Mach number air stream. International Journal of Multiphase Flow $32(1), 20-50$.

Harlow, F. H., Amsden, A. A., Oct. 1971. A numerical fluid dynamics calculation ${ }_{445}$ method for all flow speeds. Journal of Computational Physics 8 (2), 197-213.

Hu, X. Y., Khoo, B. C., Jul. 2004. An interface interaction method for compressible multifluids. Journal of Computational Physics 198 (1), 35-64.

Hu, Z. Z., Mai, T., Greaves, D., Raby, A., Nov. 2017. Investigations of offshore breaking wave impacts on a large offshore structure. Journal of Fluids and Structures 75 (Supplement C), 99-116.

Huber, G., Tanguy, S., Béra, J.-C., Gilles, B., Dec. 2015. A time splitting projection scheme for compressible two-phase flows. Application to the interaction of bubbles with ultrasound waves. Journal of Computational Physics 302 (Supplement C), 439-468.

455 Kadioglu, S. Y., Sussman, M., Osher, S., Wright, J. P., Kang, M., Nov. 2005. A second order primitive preconditioner for solving all speed multi-phase flows. Journal of Computational Physics 209 (2), 477-503.

Kang, M., Fedkiw, R. P., Liu, X.-D., Sep. 2000. A Boundary Condition Capturing Method for Multiphase Incompressible Flow. Journal of Scientific Computing 15 (3), 323-360. 
Knudsen, E., Doran, E. M., Mittal, V., Meng, J., Spurlock, W., Jan. 2017.

Compressible Eulerian needle-to-target large eddy simulations of a diesel fuel injector. Proceedings of the Combustion Institute 36 (2), 2459-2466.

Koren, B., Lewis, M. R., van Brummelen, E. H., van Leer, B., Sep. 2002. Riemann-Problem and Level-Set Approaches for Homentropic Two-Fluid Flow Computations. Journal of Computational Physics 181 (2), 654-674.

Kuila, S., Raja Sekhar, T., Zeidan, D., Aug. 2015. A Robust and accurate Riemann solver for a compressible two-phase flow model. Applied Mathematics and Computation 265 (Supplement C), 681-695.

470 Kwatra, N., Su, J., Grétarsson, J. T., Fedkiw, R., Jun. 2009. A method for avoiding the acoustic time step restriction in compressible flow. Journal of Computational Physics 228 (11), 4146-4161.

Ma, Z. H., Causon, D. M., Qian, L., Mingham, C. G., Martínez Ferrer, P., Sep. 2016. Numerical investigation of air enclosed wave impacts in a depressurised tank. Ocean Engineering 123 (Supplement C), 15-27.

Miller, S. T., Jasak, H., Boger, D. A., Paterson, E. G., Nedungadi, A., Oct. 2013. A pressure-based, compressible, two-phase flow finite volume method for underwater explosions. Computers \& Fluids 87 (Supplement C), 132-143.

Ménard, T., Tanguy, S., Berlemont, A., May 2007. Coupling level set/VOF/ghost fluid methods: Validation and application to 3d simulation of the primary break-up of a liquid jet. International Journal of Multiphase Flow 33 (5), 510-524.

Perigaud, G., Saurel, R., Oct. 2005. A compressible flow model with capillary effects. Journal of Computational Physics 209 (1), 139-178.

${ }_{485}$ Petitpas, F., Massoni, J., Saurel, R., Lapebie, E., Munier, L., Aug. 2009. Diffuse interface model for high speed cavitating underwater systems. International Journal of Multiphase Flow 35 (8), 747-759. 
Rudman, M., Aug. 1998. A volume-tracking method for incompressible multifluid flows with large density variations. International Journal for Numerical Methods in Fluids 28 (2), 357-378.

Saurel, R., Abgrall, R., Apr. 1999. A Multiphase Godunov Method for Compressible Multifluid and Multiphase Flows. Journal of Computational Physics $150(2), 425-467$.

Schochet, S., May 2005. The mathematical theory of low Mach number flows. ESAIM: Mathematical Modelling and Numerical Analysis 39 (3), 441-458.

Shu, C.-W., Nov. 1997. Essentially non-oscillatory and weighted essentially nonoscillatory schemes for hyperbolic conservation laws. Tech. Rep. NASA CR97-206253 ICASE Report No. 97-65, Institute for Computer Applications in Science and Engineering.

Sussman, M., Smith, K. M., Hussaini, M. Y., Ohta, M., Zhi-Wei, R., Feb. 2007. A sharp interface method for incompressible two-phase flows. Journal of Computational Physics 221 (2), 469-505.

Tanguy, S., Ménard, T., Berlemont, A., 2007. A Level Set Method for vaporizing two-phase flows. Journal of Computational Physics 221 (2), 837 - 853.

Tharakan, T. J., Ramamurthi, K., 2010. Influence of liquid and gas compressibility on the growth of waves in thin liquid sheets. Fluid Dyn. Res. 42 (3), 035508 .

Vaudor, G., Ménard, T., Aniszewski, W., Doring, M., Berlemont, A., Jul. 2017. A consistent mass and momentum flux computation method for two phase flows. Application to atomization process. Computers \& Fluids 152 (Supplement C), 204-216.

Zhou, Z. W., Lin, S. P., Jul. 1992. Effects of compressibility on the atomization of liquid jets. Journal of Propulsion and Power 8 (4), 736-740. 